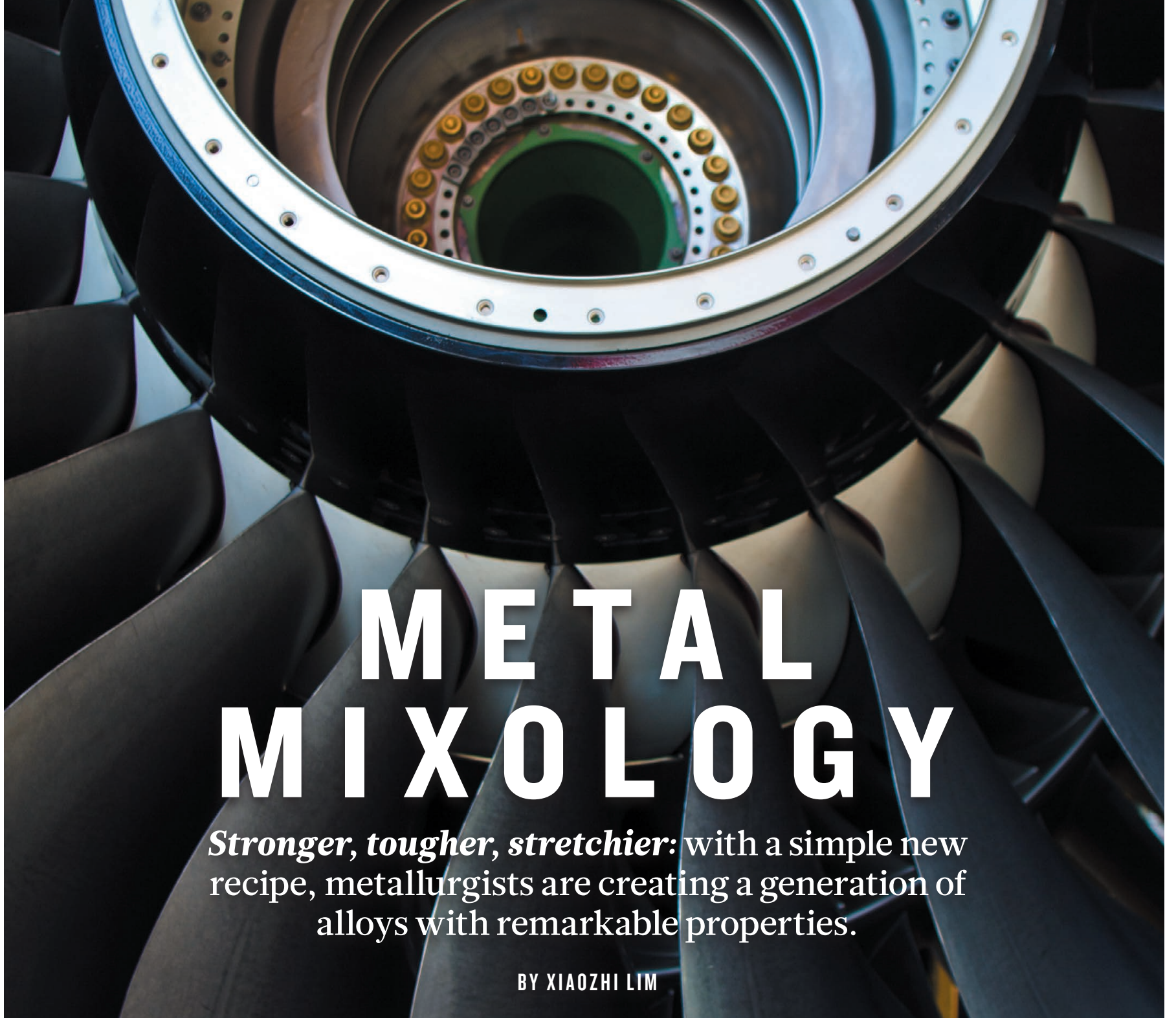

$\mathrm{t}$ first glance, the machine seems to be building a miniature cityscape. A ring of nozzles fires four jets of powdered metal into a downward-pointed laser beam, which fuses the colliding grains in a bright orange glow. The mixed grains then solidify on the growing tip of a small pillar of metal alloy. Once the pillar is 1-2 centimetres high, the platform that holds it shifts to the side, and the machine starts to build another one right next door. The result looks like a forest of toy skyscrapers.

In reality, these towers, generated at the Ames Laboratory in Iowa, reflect a major shift in how researchers think about alloys. The standard recipe - used for technologies ranging from ancient swords and arrowheads to modern jet-engine turbines - is to take a useful metal and mix in a pinch of this or a touch of that to improve its properties. One classic example is the addition of carbon to iron to make steel.

But the machine at Ames is making experimental samples of 'highentropy' alloys, which consist of four, five or more elements mixed together in roughly equal ratios. This deceptively simple recipe can yield alloys that are lighter and stronger than their conventional counterparts, while being much more resistant to corrosion, radiation or severe wear. Eventually, researchers hope, this approach could even produce alloys that have magnetic or electrical properties never seen before, leading to whole new generations of technology.

"We have almost explored everything for traditional alloys," says Yong Zhang, a materials scientist with the State Key Laboratory for Advanced Metals and Materials at the University of Science and Technology Beijing. "For high-entropy alloys, the science is very new," he says - so new that no such alloy has yet made the leap from lab to market. But some researchers are working to make that happen, eyeing potential applications that range from high-temperature furnace linings to ultralightweight aerospace materials. And the field has attracted funding from research agencies in China, Europe, the United States and elsewhere.

"We're not talking about a narrow class of materials, but an extremely broad philosophy on how to combine elements," says Daniel Miracle, a materials scientist at the Air Force Research Laboratory at the WrightPatterson Air Force Base in Ohio. "The opportunity to find something new and exciting is very high." Last year, he and his colleagues estimated $^{1}$ that almost 313,560 different alloys can be made by combining exactly equal proportions of 3, 4, 5 or 6 metallic elements from a set of just 26. More possibilities can come from varying the proportions or expanding the choice of elements.

But not every combination is a winner, says Easo George, a materials engineer at Ruhr University Bochum in Germany. Scientists are still learning what works and what doesn't. Still, he says, "the space available for exploration is really huge, and we have only looked at a small portion of the Universe".

The idea for high-entropy alloys first struck metallurgist Jien-Wei Yeh in 1995, while he was driving through the Taiwanese countryside. The physics of conventional alloys was already well understood, says Yeh, who works at National Tsing Hua University in Hsinchu, Taiwan. At the atomic level, pure metals have a regular crystal structure that stacks layer upon layer of identical atoms. Often, these layers slip past each other easily, which makes the metal too soft to be useful. That is 
why pure gold is rarely used in jewellery: it cannot survive much wear and tear. But if a metalsmith mixes in an element with a different atomic size, the interloper will randomly disrupt the layers and reduce their tendency to slip, which creates a much harder alloy. The correct choice of compounds can enable metallurgists to tailor other properties as well, such as corrosion resistance or melting point.

But Yeh was also well aware of the potential complications. If too much of the alloying element were added, for example, its atoms could stop falling randomly among the layers, and instead might start to alternate with the primary metal atoms in a more regular pattern, producing a compound that is weak and brittle.

And that gave him an idea, says Yeh: instead of starting with one primary material and mixing in small quantities of one or two elements, why not stir together similar quantities of four or five elements - or even more? The number of possible ways for the different atoms to arrange themselves would expand dramatically, leading to a tendency towards disorder, or 'high entropy', that would overwhelm any bias favouring a regular crystallattice structure. Because each of the randomly mixed elements would be a different size, the atoms would become lodged into place and less able to slide past each other, creating a very hard material (see 'Tough and strong').

It was a strange idea at the time, and Yeh gave it a low priority even in his own laboratory; it wasn't until 2004 that his research group first reported success at mixing five to ten elements together ${ }^{2}$, producing alloys that were considerably harder than stainless steel. Another group independently announced similar findings around the same time ${ }^{3}$.

\section{SPOILT FOR CHOICE}

The field began to move rapidly after that. In 2009, Zhang described ${ }^{4}$ an alloy composed of cobalt, chromium, copper, iron, nickel and aluminium that was more than 14 times stronger than pure aluminium, but had nearly 3 times the ductility - a measure of a metal's ability to stretch without breaking. In 2011, Yeh devised ${ }^{5}$ a cobalt, chromium, iron, nickel, aluminium and titanium alloy that is twice as resistant to damage from friction as conventional wear-resistant steels. And in 2014, George and his team concocted ${ }^{6}$ a cobalt, chromium, iron, manganese and nickel alloy that can be cooled below liquid-nitrogen temperatures without becoming brittle. The material could be useful for cryogenic vessels, natural-gas pipelines and other low-temperature applications, such as spacecraft.

Often, however, the best thing about high-entropy alloys - the massive number of possibilities - can also be researchers' biggest challenge. With more than 80 metallic elements in the periodic table, says Miracle, "there are way too many alloys to test, and not enough time". For his own work on high-entropy alloys for aircraft engines and aeroplane frames, he is searching for materials that are lighter, more resistant to corrosion and better able to maintain their strength at high temperatures than anything currently available. To cope with the abundance of choices, Miracle is focusing on elements such as niobium, tantalum and chromium, which have high melting points to begin with.

Another strategy is to try to replicate the characteristics of alloys that are known to work well. For example, some steels are not just a random mix of atoms, but instead contain small nodules of compounds that form when the steels cool rapidly. Although such a composite structure is less stable than a random mixture, it confers high ductility to the steels. Cem Tasan, a metallurgist at the Massachusetts Institute of Technology in Cambridge, has used that knowledge to blend iron, manganese, cobalt and chromium into a high-entropy alloy ${ }^{7}$ that is simultaneously extremely hard and highly ductile - traits that once seemed completely incompatible. "It doesn't make sense to abandon all that we know," he says.

The mini-skyscrapers at Ames represent another, more systematic approach. The machine can build up to 30 pillars in less than an hour with a slightly different mix of raw materials in each, so that researchers can test the properties of many alloys quickly. Matthew Kramer, a materials scientist at Ames, leads a project to find high-entropy alloys that can withstand high temperatures and resist corrosion, which could help power plants to operate at higher temperatures and become more efficient.

Aiding his team is Duane Johnson, a theorist at Ames who, in 1995, developed an algorithm to predict the properties of conventional alloys before they are made ${ }^{8}$. In 2015, he expanded the code to work for high-entropy alloys ${ }^{9}$. Johnson's algorithm assesses how much one element is attracted to or repelled by another, and then using that information to predict whether a mixture of elements will form a compound, a solid solution or a mixture of both. That enables Kramer's team to identify which alloys might be worth investigating. The experimental results are then fed back into the algorithm to validate and improve the code.

There are a number of obstacles to overcome to move the highentropy-alloy field forward. So far, the emphasis has been on improving structural properties, such as strength. But there has been much less work on developing alloys with specific 'functional' properties, including conductivity or response to a magnetic field - a development that would enable applications in areas such as refrigeration and electronics.

Nonetheless, there are many possibilities still to be explored, especially as researchers start to extend the concept well beyond its original definition. Zhang, for example, is mixing metals with elements such as carbon, nitrogen and silicon in an effort to develop new high-temperature ceramics for use in solar-power applications.

And some, including Tasan and Yeh, have begun to experiment with alloys that contain elements mixed in high, but unequal proportions. Their preliminary findings show that many of these still have all the properties that make high-entropy alloys desirable in the first place. For example, Yeh has prepared a range of hard materials that consist of $50 \%$ nitrogen, carbon or oxygen, along with a mix of other elements, such as aluminium, silicon or titanium, that can withstand scratching. They could be used as long-lasting coatings for machine parts and cutting tools. "We now have this rich, rich field for exploration," says George.

XiaoZhi Lim is a freelance writer based in Singapore.

1. Senkov, O. N., Miller, J. D., Miracle, D. B. \& Woodward, C. Nature Commun. 6, 6529 (2015).

2. Yeh, J.-W. et al. Adv. Eng. Mater. 6, 299-303 (2004)

3. Cantor, B., Chang, I. T. H., Knight, P. \& Vincent, A. J. B. Mater. Sci. Eng. A 375-377, 213-218 (2004).

4. Wang, F., Zhang, Y., Chen, G. \& Davies, H. A. Int. J. Mod. Phys. B 23, 1254-1259 (2009).

5. Chuang, M.-H., Tsai, M.-H., Wang, W.-R., Lin, S.-J. \& Yeh, J.-W. Acta Mater. 59, 6308-6317 (2011).

6. Gludovatz, B. et al. Science 345, 1153-1158 (2014)

7. Li, Z., Pradeep, K. G., Deng, Y., Raabe, D. \& Tasan, C. C. Nature http://dx.doi. org/10.1038/nature17981 (2016).

8. Althoff, J.D., Johnson, D.D. \& F. J. Pinski Phys. Rev. Lett. 74, 138 (1995)

9. Singh, P., Smirnov, A. V. \& Johnson, D. D. Phys. Rev. B 91, 224204 (2015). 